\title{
Atypical meningiomas: is postoperative radiotherapy indicated?
}

\author{
Kangmin D. Lee, M.D., ${ }^{1,5}$ John J. DePowell, M.D., ${ }^{1}$ Ellen L. Air, M.D., Ph.D. ${ }^{1,3,4}$ \\ Alok K. Dwivedi, Ph.D., ${ }^{6}$ Ady Kendler, M.D., ${ }^{2}$ and Christopher M. McPherson, M.D. 1,3,4 \\ Departments of ${ }^{1}$ Neurosurgery and ${ }^{2}$ Pathology and Laboratory Medicine, University of Cincinnati College of \\ Medicine; ${ }^{3}$ Brain Tumor Center at UC Neuroscience Institute; and ${ }^{4}$ Mayfield Clinic, Cincinnati, Ohio; \\ ${ }^{5}$ Department of Neurosurgery, Hackensack University Medical Center, Hackensack, New Jersey; and \\ ${ }^{6}$ Division of Biostatistics \& Epidemiology, Texas Tech University Health Sciences Center, El Paso, Texas
}

\begin{abstract}
Object. The role of postoperative radiation therapy after surgery for atypical meningiomas remains controversial. In this retrospective cohort study, the authors examine the recurrence rates for atypical meningiomas after resection (with or without adjuvant radiotherapy) and identify which factors were associated with recurrence.

Methods. Of 90 patients with atypical meningiomas who underwent surgery between 1999 and 2009, 71 (79\%) underwent gross-total resection (GTR) and 19 (21\%) underwent subtotal resection (STR); 31 patients received adjuvant radiotherapy. All tumors were pathology-confirmed WHO Grade II atypical meningiomas. Univariate and multivariate analyses were performed to identify factors associated with recurrence-free survival.

Results. Among 90 patients, 17 developed tumor recurrence ( $81 \%$ recurrence-free survival at 5 years). In the overall group, adjuvant radiotherapy reduced the recurrence rate to $9 \%$ from $19 \%$ at 5 years $(\mathrm{p}=0.048)$. After STR, adjuvant radiotherapy significantly reduced recurrence from $91 \%$ to $20 \%(\mathrm{p}=0.0016)$. However, after GTR, adjuvant radiotherapy did not significantly reduce the recurrence rate $(16.7 \%$ without radiation therapy vs $11.8 \%$ with radiation therapy) $(\mathrm{p}=1.00)$. Five factors independently predictive of tumor recurrence included mitotic index, sheeting, necrosis, nonuse of radiation therapy, and STR. Further recursive partitioning analysis showed significant increases in risk for patients older than 55 years with mitoses and sheeting.

Conclusions. Adjuvant radiotherapy was effective at lowering recurrence rates in patients after STR but delivered no significant improvement in patients after GTR. Given that rates after GTR were similar with or without adjuvant radiotherapy, close observation without postoperative radiation therapy may be a viable option for these patients. Patients older than 55 years and those with mitoses noted during pathological examination had a significant risk of recurrence after GTR; for these patients, postoperative radiotherapy is recommended. (http://thejns.org/doi/abs/10.3171/2013.9.FOCUS13325)
\end{abstract}

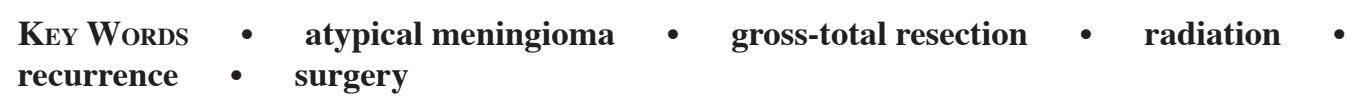

$\mathrm{M}$ ENINGIOMAS are the most common type of primary brain tumor and represent $20 \%$ of all intracranial tumors. ${ }^{1,2}$ Although the majority of meningiomas are benign, $5 \%-23 \%$ are classified as atypical and are associated with an increased risk of recurrence. ${ }^{19,17}$ While the recognition and definition of this atypical subclass is in ongoing transition, its incidence appears to be increasing. ${ }^{17}$ The latest WHO guidelines (2007) categorize atypical meningiomas by the following features: increased mitotic activity ( $\geq 4$ mitotic figures per high-power field) or 3 or more of the following histological features: 1) loss of lobular architecture (sheeting), 2) prominent nucleoli, 3) hypercellularity, 4) small cells with high nuclear to cytoplasmic ratios, and 5) foci of spontaneous necrosis.

Abbreviations used in this paper: GTR = gross-total resection; $\mathrm{RPA}=$ recursive partitioning analysis; STR $=$ subtotal resection.
Regardless of the tumor grade, resection is one of the primary treatment options for all meningiomas. Benign meningiomas lack aggressive features and have a low mitotic rate and slow growth. ${ }^{12,13}$ The low recurrence rates for Grade I meningiomas after gross-total resection (GTR) obviate the need for postoperative radiation therapy.,16 After subtotal resection (STR), some authors recommend postoperative irradiation, even for these Grade I tumors, ${ }^{4}$ whereas others advocate a wait-and-see approach. The higher recurrence rates for Grade III anaplastic meningiomas lead most practitioners to recommend radiation therapy after resection, whether GTR or STR.?

The role of postoperative radiation therapy remains a controversial issue for Grade II atypical meningiomas. Surveying neurosurgeons in the United Kingdom and the Republic of Ireland regarding treatment for atypical meningiomas, Marcus et al. found that $80 \%$ of respondents would not advocate radiation therapy after GTR and $41 \%$ would not advocate radiotherapy even after STR. ${ }^{8}$ In a 
sampling of 56 centers in Germany, a minority (16\%) of institutions routinely used radiotherapy after GTR of atypical meningiomas. ${ }^{15}$

To better address this problem and develop a treatment paradigm for postoperative management of atypical meningiomas, we retrospectively reviewed our 10-year surgical experience by comparing the rates of tumor recurrence after STR or GTR and by examining the role of postoperative radiotherapy in prevention of recurrence. Finally, we identified prognostic factors associated with recurrence in hopes of refining future management.

\section{Methods}

\section{Patient Selection}

In this retrospective cohort study of 779 patients who underwent resection of intracranial meningiomas at the University of Cincinnati Medical Center between 1999 and 2009, 90 men and women had pathology-confirmed Grade II atypical meningiomas and at least 1 year of follow-up. Extent of resection was based on both the surgeon's impression during surgery and our review of the first postoperative MRI scans. All pathology slides were reviewed or re-reviewed by a single neuropathologist (A.K.), and all cases were confirmed as Grade II atypical meningioma by WHO 2007 criteria. All patients who received radiation therapy postoperatively underwent fractionated stereotactic radiotherapy by linear accelerator (median dose 59.4 Gy, range 50.4-60.0 Gy) delivered to the tumor bed in 1.8- to 2.0-Gy fractions. This study was approved by the institutional review board at the University of Cincinnati.

\section{Parameters Assessed}

Tumor size was determined by evaluation of the preoperative MR images, measuring along the longest axis and rounded to the nearest millimeter. Tumor locations were classified into one of 5 categories: convexity, falx/ parasagittal, sphenoid wing, midline anterior skull base, or other. Simpson Grade I-III resections were considered GTRs, whereas Grade IV resections were considered STRs. In determining which factors were associated with tumor recurrence, 14 possible variables were included in the analysis: 1) patient age, 2) sex, 3) tumor location (falx/parasagittal, midline anterior skull base, others, and convexity), 4) tumor size, 5) MIB-1, 6) mitotic index, 7) hypercellularity, 8) small cells, 9) sheeting, 10) prominent nucleoli, 11) necrosis, 12) brain invasion, 13) radiation therapy, and 14) extent of resection as STR or GTR.

\section{Statistical Analysis}

Quantitative variables were described using mean, standard deviation, and range, and nonnormal quantitative variables were described using median and range. Categorical variables were described using frequency and percentage. Recurrence-free survival was measured from the date of surgery to either the date of recurrence for patients after GTR or progression after STR. Patients who had no recurrences at the end of follow-up were considered as censored. Overall survival was measured from the date of surgery to death. Patients who did not die at the end of follow-up were considered as censored. Median survival at 1,2, and 5 years by $95 \%$ CIs were calculated for recurrence-free and overall survival. The log-rank test was used to compare the recurrence-free survival according to categorized factors.

Factors associated with recurrence were assessed using univariate and multivariate Cox proportional hazards model. Proportionality assumption was assessed using the Schoenfeld test. The significant cofactors at $10 \%$ level of significance under univariate analysis were included in stepwise multivariate Cox analysis. Results of Cox analysis were reported using HRs with 95\% CIs and $p$ values. Kaplan-Meier curves were constructed for rates of overall survival solely and for the combined overall survival and recurrence-free survivals; these curves were constructed according to treatment groups. Recursive partitioning analysis (RPA) was performed for time to recurrence outcome using the "rpart" package (R 2.12.1). All $p$ values $\leq$ 0.05 were regarded as significant. Other statistical analyses were conducted using STAT version 12.1 and SAS version 9.3.

\section{Results}

A total of 90 patients ( 34 men and 56 women; mean age $56.9 \pm 13.4$ years, range $22-83$ years) with pathology-confirmed Grade II atypical meningiomas underwent resection (Table 1). The most common tumor locations were convexity (47.8\%) followed by falx/parasagittal (21.1\%). Tumor size averaged $4.8 \pm 1.5 \mathrm{~cm}$ (Table 2). Patients underwent follow-up for a median of 48.7 months (range 12-108 months); more than half of the patients

TABLE 1: Characteristics of patients who underwent surgical resection for atypical meningioma*

\begin{tabular}{ll}
\hline \multicolumn{1}{c}{ Characteristic } & \multicolumn{1}{c}{ Value } \\
\hline no. of patients & 90 \\
mean age \pm SD in yrs & $56.9 \pm 13.4$ \\
median follow-up in mos & 48.7 \\
no. of patients who received adjuvant XRT & $31(34.4)$ \\
no. of recurrences & $17(18.9)$ \\
median time to recurrence in mos & 31 \\
surgery & \\
$\quad$ GTR (Simpson Grade I-III) & $71(78.9)$ \\
$\quad$ STR (Simpson Grade IV) & $19(21.1)$ \\
preop XRT & \\
$\quad$ GTR (Simpson Grade I-III) & $17 / 71(23.9)$ \\
STR (Simpson Grade IV) & $14 / 19(73.7)$ \\
recurrence rate & \\
$\quad$ GTR+XRT & $2 / 17(11.8)$ \\
GTR w/o XRT & $9 / 54(16.7)$ \\
STR+XRT & $1 / 14(7.1)$ \\
STR w/o XRT & $5 / 5(100)$ \\
\hline * Values are the number of patients (\%) unless noted otherwise. XRT \\
* radiation therapy.
\end{tabular}


Atypical meningiomas

TABLE 2: Tumor location, size, and recurrences for 90 atypical meningiomas

\begin{tabular}{lccc}
\hline \multicolumn{1}{c}{ Location } & No. of Patients $(\%)$ & Mean Diameter $(\mathrm{cm})$ & No. of Recurrences $(\%)$ \\
\hline all locations & 90 & 4.8 & $17(19)$ \\
convexity & $43(47.8)$ & 4.9 & $6(14)$ \\
falx/parasagittal & $19(21.1)$ & 4.7 & $5(26.3)$ \\
sphenoid wing & $8(8.9)$ & 5.1 & $0(0.0)$ \\
midline anterior skull base & $7(7.8)$ & 4.5 & $2(28.6)$ \\
other & $13(14.4)$ & 4.6 & $4(30.8)$ \\
\hline
\end{tabular}

(52\%) underwent follow-up either to recurrence or for at least 4 years after resection.

The overall survival for our 90 patients after resection was $96 \%$ at 5 years (Fig. 1); 17 (19\%) of these patients had tumor recurrence on average within 31 months (range 7-82 months). Recurrence-free survival rates were $96.7 \%$ at 1 year, $91.3 \%$ at 2 years, and $81.1 \%$ at 5 years.

Of the 71 tumor resections $(79 \%)$ categorized as Simpson Grade I-III resections and deemed GTR, 17 patients $(24 \%)$ received postoperative adjuvant radiotherapy. Recurrences developed in 2 (12\%) of these 17 patients and in 9 patients (17\%) without radiation therapy; the 5-year recurrence-free survivals were $74 \%$ and $65 \%$, respectively, and this difference was not statistically significant $(\mathrm{p}=1.00)$.

Of the 19 patients undergoing Simpson Grade IV tumor resections, 14 (74\%) received adjuvant postoperative radiotherapy. Of these 14 patients, only $1(7 \%)$ patient developed a recurrence at 22 months. Recurrences developed in 5 patients without radiation therapy (91\% vs $20 \%$ recurrence-free survival at 5 years, respectively); this difference was statistically significant $(p=0.0016)$.

\section{Factors Associated With Tumor Recurrence}

In unadjusted analysis, MIB-1, mitotic index, sheeting, necrosis, and STR were found to be associated with recurrence-free survival, whereas age and radiation therapy were borderline significant (Table 3). In multivariate analysis, the 5 factors independently predictive of tumor recurrence included mitotic index (HR 1.24, $\mathrm{p}=0.004)$, sheeting (HR 6.55, p = 0.002), necrosis (HR 6.05, $\mathrm{p}=$ $0.004)$, lack of radiation therapy (HR 0.02, p = 0.0001), and STR (HR 5.31, p = 0.007) (Table 4). Subgroup analysis revealed that adjuvant radiation more significantly reduced the risk of tumor recurrence in the STR population ( $\mathrm{p}=0.0016$, Bonferroni adjusted log-rank test). In evaluating the extent of resection on tumor recurrence, we found that patients fared better after GTR than STR (Fig. 1).

An RPA was performed to identify which factors make patients most susceptible to recurrence (Fig. 2). The HR of recurrence was 1.61 for older patients $(>55$ years) - a rate that increased to 2.98 when these patients showed evidence of mitotic activity on tumor histology. Finally, this risk was highest at 3.66 for patients older than 55 years who had both mitotic activity and sheeting on tumor histology.

\section{Discussion}

In our retrospective cohort study of recurrence rates after resection in 90 patients, representing one of the largest series of surgically treated atypical meningiomas to date, postoperative radiation therapy effectively lowered the risk of recurrence after STR but not after GTR. In multivariate analysis of 14 factors that could affect recurrence, 5 factors independently predictive included mitotic index, sheeting, necrosis, radiation therapy, and STR. Furthermore, RPA of patients showed a significant increase in the risk of recurrence for patients older than 55 years with mitoses and with sheeting.

The clinical care of patients with atypical meningiomas remains controversial and is a pressing problem for clinicians. Since the implementation of the 2000 WHO grading changes, the incidence for these tumors has been increasing. In our study of 779 meningiomas, 120 tumors $(15.4 \%)$ were graded as atypical-a rate similar to incidence data from other published series that range from $4.7 \%$ to $23 \%$. ${ }^{1,9,17,18}$

As pathology grading continues to change, earlier studies are less relevant. Our study is strengthened by the fact that all cases were reviewed by a single neuropathologist, who then re-reviewed all pathology slides according to the 2007 WHO guidelines for tumor grading. In a retrospective audit of meningioma grading performed in the United Kingdom using pre- and post-2000 WHO guidelines, Willis et al. noted a $20.4 \%$ incidence rate for atypical meningiomas; importantly, $38 \%$ of the Grade II meningiomas had originally been classified as Grade I using the pre-2000 WHO guidelines. ${ }^{19}$ In another retrospective audit, Smith et al. reported atypical meningioma grading increased from $18 \%$ to $23 \% .{ }^{17}$ However, the limitations of earlier studies were the combination of both atypical and anaplastic meningiomas and mixture of both pre- and post-2000 WHO guidelines.,5,10,11 Based on these inconsistencies, drawing concrete conclusions is difficult regarding the role of different therapies in tumor recurrence for atypical meningiomas.

\section{Atypical Meningioma Recurrence}

Resection has always been and remains one of the most important treatment modalities for meningiomas, whether WHO Grade I, II, or III. Multivariate analysis in this study confirmed that extent of resection was particularly important; this finding concurred with other studies that indicate extent of resection is a strong prog- 
K. D. Lee et al.
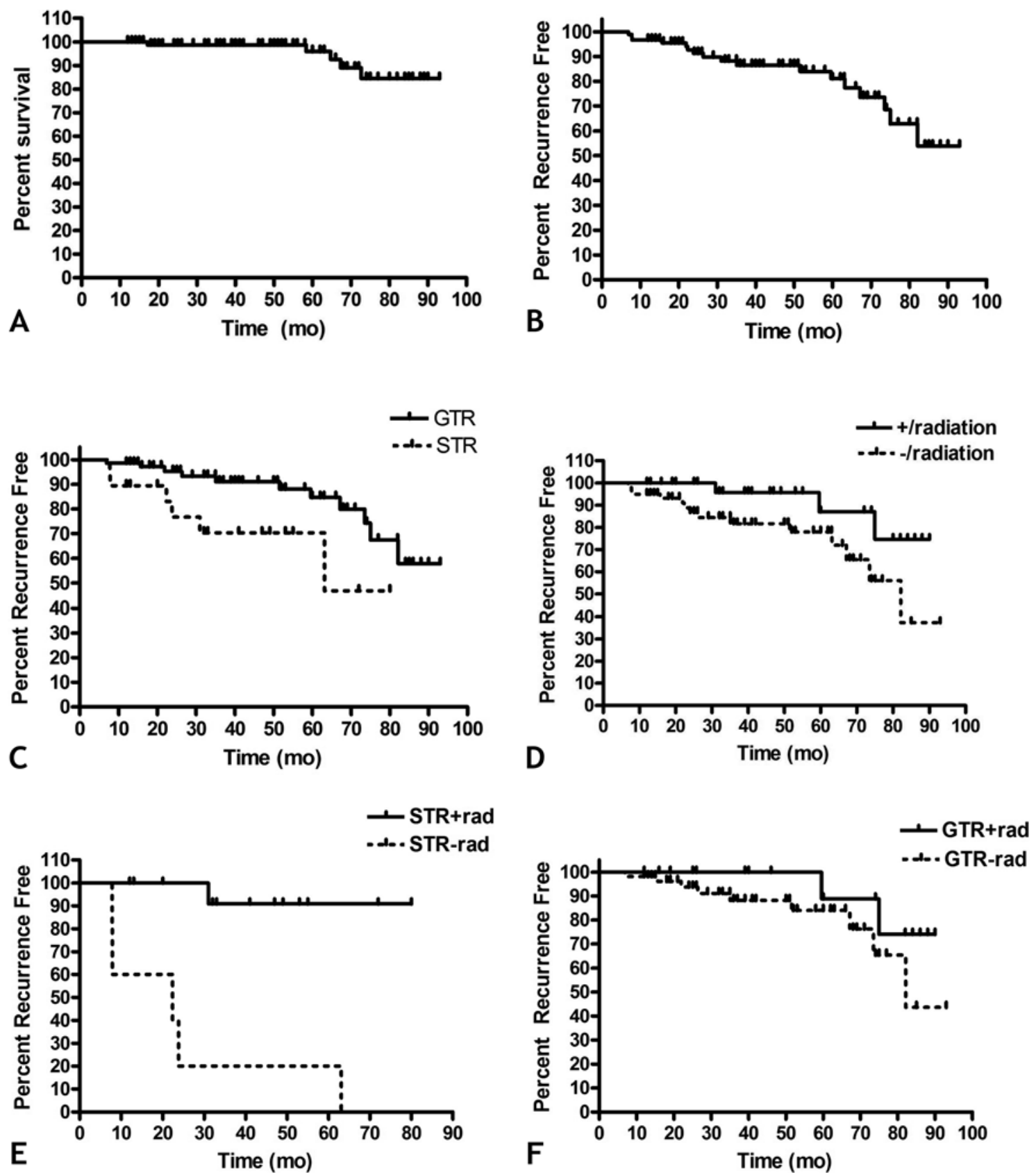

FIG. 1. Kaplan-Meier curves. A: Overall survival for 90 patients who underwent resection of an atypical meningioma. B-F: Recurrence-free survival comparisons for the overall group $(B)$, after GTR or STR $(p=0.03)(C)$, for the overall group with or without radiation therapy $(p=0.04)(D)$, STR group with or without radiation therapy $(p=1.00)(E)$, and GTR group with or without radiation therapy $(p=0.0016)(F)$.

nostic factor for tumor recurrence in all grades of meningiomas. $3,10,14,16$ The importance of extent of resection is further reinforced by the proclivity for Grade II meningiomas to occur in areas where GTR is often easier to achieve. Most of the atypical meningiomas in our patients occurred in the convexity (48\%) - a location where most $(79 \%)$ could then undergo complete resection.

Although the importance of extent of resection appears clear in reducing the risk of recurrence, consensus is lacking, even in the most recent reports, regarding the role of adjuvant radiation after resection. In a 2009 study,
Aghi et al. found a high rate of recurrence (41\%) for atypical meningiomas 5 years after GTR; although their data did not show a statistically significant effect, the authors ultimately found at least a suggested benefit for postoperative radiation in tumors after GTR. ${ }^{1}$ In a 2011 study evaluating the role of postoperative radiotherapy for 114 patients after resection for atypical meningiomas, Mair et al. found that neither radiotherapy nor extent of resection statistically impacted recurrence for the entire group; these authors then recommended against postoperative radiotherapy for patients after GTR of their tumors.? 
TABLE 3: Univariate Cox proportional hazards analysis: HRs of patient-related and histological factors in predicting recurrence of resected atypical meningiomas

\begin{tabular}{lcc}
\hline \multicolumn{1}{c}{ Variable } & $\mathrm{HR}(95 \% \mathrm{Cl})$ & $\mathrm{p} \mathrm{Value}$ \\
\hline age (yrs) & $1.04(1.00-1.08)$ & 0.06 \\
female sex & $0.75(0.29-1.94)$ & 0.55 \\
tumor size (cm) & $1.00(0.96-1.03)$ & 0.78 \\
tumor location & & \\
$\quad$ falx/parasagittal & $2.37(0.71-7.88)$ & 0.16 \\
$\quad$ midline anterior skull base & $1.33(0.26-6.71)$ & 0.73 \\
$\quad$ others & $1.39(0.39-4.94)$ & 0.61 \\
$\quad$ convexity (referent) & & \\
MIB-1 & $1.06(1.01-1.12)$ & 0.03 \\
mitotic index & $1.22(1.07-1.39)$ & 0.002 \\
hypercellularity & $1.59(0.36-6.99)$ & 0.54 \\
small cells & $1.49(0.55-4.05)$ & 0.43 \\
sheeting & $2.73(1.03-7.26)$ & 0.04 \\
prominent nucleoli & $1.80(0.69-4.72)$ & 0.23 \\
necrosis & $3.30(1.26-8.62)$ & 0.01 \\
brain invasion & $0.64(0.18-2.25)$ & 0.48 \\
adjuvant XRT & $0.30(0.09-1.06)$ & 0.06 \\
STR & $3.15(1.13-8.84)$ & 0.03 \\
\hline
\end{tabular}

In our series, adjuvant radiation was administered after initial surgery to 31 patients, a segment that represented a large proportion of our study population. Therefore, our subgroup analysis did reach statistical significancean analysis that has been lacking in previous studies. Although radiotherapy significantly decreased the risk of recurrence among patients after STR, there was no statistical effect after GTR. Therefore, we advocate that, instead of routine radiation therapy, close observation after
TABLE 4: Multivariate Cox proportional hazards analysis: HRs of patient-related and histological factors in predicting recurrence of resected atypical meningiomas

\begin{tabular}{lll}
\hline \multicolumn{1}{c}{ Variable } & \multicolumn{1}{c}{$\mathrm{HR}(95 \% \mathrm{Cl})$} & $\mathrm{p}$ Value \\
\hline mitotic index & $1.24(1.07-1.44)$ & 0.004 \\
sheeting & $6.55(1.95-21.99)$ & 0.002 \\
necrosis & $6.05(1.81-20.20)$ & 0.004 \\
no adjuvant XRT & $0.02(0.00-0.12)$ & 0.0001 \\
STR & $5.31(1.58-17.85)$ & 0.007 \\
\hline
\end{tabular}

GTR for atypical meningiomas may be a potential therapeutic option. This also reserves the potential for other modalities, such as stereotactic radiosurgery, if and when there is a recurrence.

\section{Study Limitations}

As a retrospective review with no formal prospective treatment plan to define which patients were treated and/ or underwent radiation therapy, selection bias may have played a role in which patients received radiation therapy, especially in the GTR group. To better define this group, we performed RPA of GTR and found that recurrences affected $45 \%$ of patients older than 55 years with any evidence of mitotic activity; therefore, for this group, we suggest postoperative radiotherapy regardless of extent of resection.

Although a significant proportion (34\%) of our patients underwent postoperative radiotherapy, no consistent protocol existed regarding the criteria for and timing of radiotherapy. Most patients did receive radiotherapy in a similar way, that is, fractionated radiation (median dose 59.4 Gy, range 50.4-60.0 Gy) delivered in 1.8- to 2.0-Gy fractions to the tumor bed. Our data suggest that radiation therapy can be withheld after GTR; however, given the

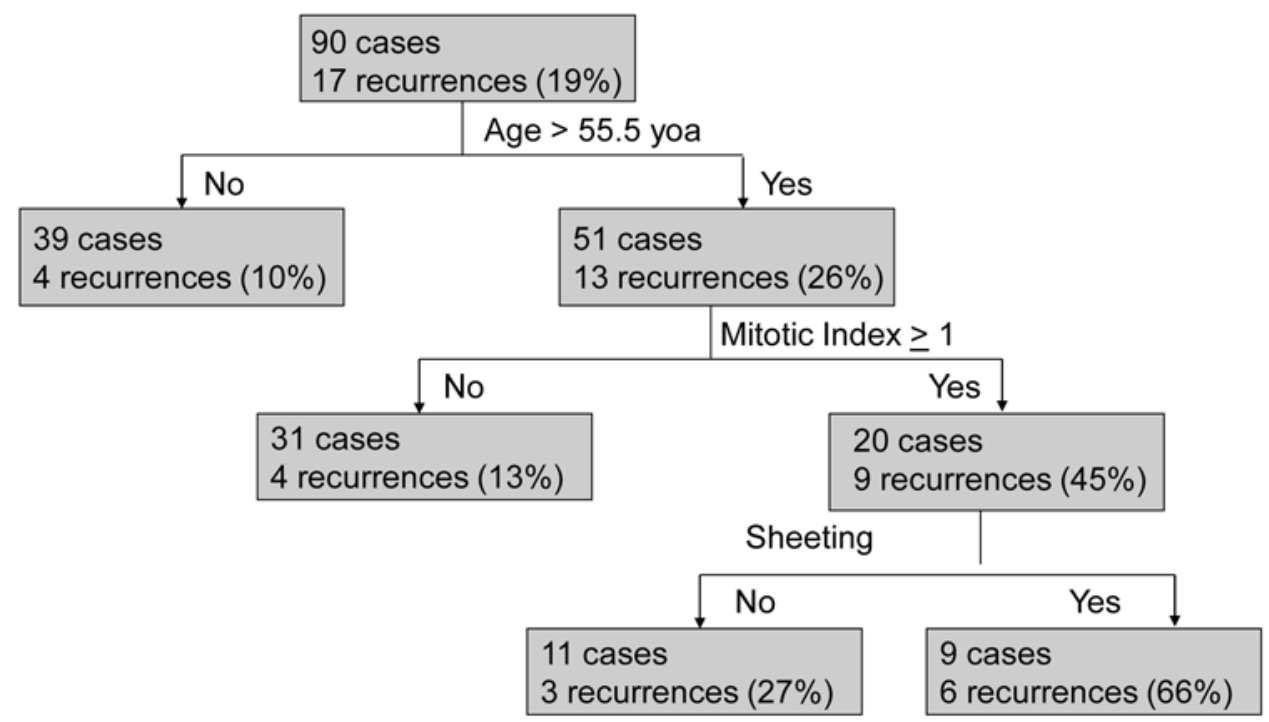

FIG. 2. Recursive partitioning analysis of 90 patients with Grade II (atypical) meningioma. The dependent variable was recurrence-free survival whereas independent variables were age, sex, tumor location, diameter, MIB-1 labeling index, mitotic index, histology, hypercellularity, presence of small cells, prominent nucleoli, sheeting, necrosis, brain invasion, resection grade, and radiotherapy. yoa = years of age. 
limitations of a retrospective study, we definitely support the importance of a randomized, prospective trial to answer this issue. Until guidelines exist based on randomized, prospective trials, we suggest that radiation therapy can be withheld until the time of recurrence for patients who undergo GTR of an atypical meningioma and who do not fit high-risk criteria as defined by our RPA analysis (that is, age $>55$ years and any mitotic activity).

In the future, more important than developing a general paradigm is to identify factors that predispose patients to suffer recurrence. Thus, the RPA performed in our study showed significantly increased recurrence in patients older than 55 years whose atypical meningiomas had evidence of any mitotic activity. Further study and research holds the potential for enabling the identification of molecular markers that could better indicate an increased risk of recurrence and need for radiation therapy, and thus facilitate a more personalized approach to these tumors.

\section{Conclusions}

The results of this study show that atypical meningiomas have a high recurrence rate after STR and that radiotherapy is beneficial in terms of decreasing that risk of recurrence. However, after GTR, the role of radiation is not as clear-there was no statistically significant benefit in terms of preventing tumor recurrence in our patients. A subgroup of patients at greater risk of recurrence was identified by RPA (age $>55$ years and evidence of mitotic activity); for these patients, given the high rate of tumor recurrence, postoperative radiation is recommended for either STR or GTR. For all other patients, close observation after GTR by serial follow-up MRI and salvage treatment at recurrence by fractionated radiotherapy or stereotactic radiosurgery or further surgery is recommended.

\section{Disclosure}

The authors report no conflict of interest concerning the materials or methods used in this study or the findings specified in this paper.

Author contributions to the study and manuscript preparation include the following. Conception and design: McPherson, Lee. Acquisition of data: McPherson, Lee, DePowell, Air, Kendler. Analysis and interpretation of data: McPherson, Lee, DePowell, Dwivedi, Kendler. Drafting the article: all authors. Critically revising the article: McPherson, Air, Dwivedi, Kendler. Reviewed submitted version of manuscript: all authors. Statistical analysis: McPherson, Lee, Dwivedi. Study supervision: McPherson.

\section{References}

1. Aghi MK, Carter BS, Cosgrove GR, Ojemann RG, AminHanjani S, Martuza RL, et al: Long-term recurrence rates of atypical meningiomas after gross total resection with or without postoperative adjuvant radiation. Neurosurgery 64:5660, 2009

2. Central Brain Tumor Registry of the United States: CBTRUS Statistical Report. Primary Brain and Central Nervous System Tumors Diagnosed in the United States in 2004-2006. (http://www.cbtrus.org/2010-NPCR-SEER/CBTRUS-WEB REPORT-Final-3-2-10.pdf) [Accessed October 15, 2013]
3. Durand A, Labrousse F, Jouvet A, Bauchet L, Kalamaridès M, Menei P, et al: WHO grade II and III meningiomas: a study of prognostic factors. J Neurooncol 95:367-375, 2009

4. Goldsmith BJ, Wara WM, Wilson CB, Larson DA: Postoperative irradiation for subtotally resected meningiomas. A retrospective analysis of 140 patients treated from 1967 to 1990 . J Neurosurg 80:195-201, 1994

5. Hug EB, Devries A, Thornton AF, Munzenride JE, Pardo FS, Hedley-Whyte ET, et al: Management of atypical and malignant meningiomas: role of high-dose, 3D-conformal radiation therapy. J Neurooncol 48:151-160, 2000

6. Ko KW, Nam DH, Kong DS, Lee JI, Park K, Kim JH: Relationship between malignant subtypes of meningioma and clinical outcome. J Clin Neurosci 14:747-753, 2007

7. Mair R, Morris K, Scott I, Carroll TA: Radiotherapy for atypical meningiomas. Clinical article. J Neurosurg 115:811-819, 2011

8. Marcus HJ, Price SJ, Wilby M, Santarius T, Kirollos RW: Radiotherapy as an adjuvant in the management of intracranial meningiomas: are we practising evidence-based medicine? $\mathbf{B r}$ J Neurosurg 22:520-528, 2008

9. Modha A, Gutin PH: Diagnosis and treatment of atypical and anaplastic meningiomas: a review. Neurosurgery 57:538550,2005

10. Palma L, Celli P, Franco C, Cervoni L, Cantore G: Long-term prognosis for atypical and malignant meningiomas: a study of 71 surgical cases. J Neurosurg 86:793-800, 1997

11. Pasquier D, Bijmolt S, Veninga T, Rezvoy N, Villa S, Krengli $\mathrm{M}$, et al: Atypical and malignant meningioma: outcome and prognostic factors in 119 irradiated patients. A multicenter, retrospective study of the Rare Cancer Network. Int J Radiat Oncol Biol Phys 71:1388-1393, 2008

12. Perry A, Scheithauer BW, Stafford SL, Lohse CM, Wollan PC: "Malignancy" in meningiomas: a clinicopathologic study of 116 patients, with grading implications. Cancer 85:20462056, 1999

13. Perry A, Stafford SL, Scheithauer BW, Suman VJ, Lohse CM: Meningioma grading: an analysis of histologic parameters. Am J Surg Pathol 21:1455-1465, 1997

14. Sanai N, Sughrue ME, Shangari G, Chung K, Berger MS, McDermott MW: Risk profile associated with convexity meningioma resection in the modern neurosurgical era. Clinical article. J Neurosurg 112:913-919, 2010

15. Simon M, Boström J, Koch P, Schramm J: Interinstitutional variance of postoperative radiotherapy and follow up for meningiomas in Germany: impact of changes of the WHO classification. J Neurol Neurosurg Psychiatry 77:767-773, 2006

16. Simpson D: The recurrence of intracranial meningiomas after surgical treatment. J Neurol Neurosurg Psychiatry 20:22-39, 1957

17. Smith SJ, Boddu S, Macarthur DC: Atypical meningiomas: WHO moved the goalposts? Br J Neurosurg 21:588-592, 2007

18. Whittle IR, Smith C, Navoo P, Collie D: Meningiomas. Lancet 363:1535-1543, 2004

19. Willis J, Smith C, Ironside JW, Erridge S, Whittle IR, Everington D: The accuracy of meningioma grading: a 10-year retrospective audit. Neuropathol Appl Neurobiol 31:141-149, 2005

Manuscript submitted August 8, 2013.

Accepted September 27, 2013.

Please include this information when citing this paper: DOI: 10.3171/2013.9.FOCUS13325.

Address correspondence to: Christopher McPherson, M.D., c/o Editorial Office, Department of Neurosurgery, University of Cincinnati College of Medicine, ML 0515, 6350 Glenway Ave., Cincinnati, OH 45267. email: editor@mayfieldclinic.com. 